\title{
Covariant Markov Dilations of Quantum Dynamical Semigroups
}

By

\author{
Alberto Frigerio*
}

\begin{abstract}
Adstract
We develop a general theory of unitary dilations of quantum dynamical semigroups, satisfying a Markov property; and we construct explicit examples by means of the noncommutative stochastic calculus of Hudson and Parthasarathy.
\end{abstract}

\section{§1. Inntrodunction}

The dilation problem for quantum dynamical semigroups is related with the question whether it is possible to interpret an irreversible evolution "without memory" of a physical system as the projection of a unitary (reversible) evolution of a larger system. The status of the problem up to 1977 was exhaustively presented by Evans and Lewis in [1]: unitary dilations were known to exist (at least at the $C^{*}$-algebraic level [2]) for all dynamical semigroups, but their physical interpretation was unclear, with the exception of the quasi-free case.

After the introduction of the notion of a quantum stochastic process $[3,4]$, it appeared that some clarification in the dilation problem was to be obtained by requiring that the dilation should define a Markov process, in some sense. After some remarks in this direction by Lewis [5], Kümmerer developed a theory of Markov dilations for completely positive (discrete and continuous) semigroups with a stationary state $([6,7]$, see also Kümmerer and Schröder [8]); in particular, the first nontrivial examples of Markov dilations for non-quasi-free semigroups were obtained. With the aid of Dümcke's theory of the singular coupling limit for multi-time correlation functions [9], Frigerio and Gorini [10] constructed stationary Markov unitary dilations for a large class of dy-

Communicated by H. Araki, December 21, 1984.

* Dipartimento di Fisica, Sezione Fisica Teorica, Università di Milano, Via Celoria 16, I-20133 Milano Italy; and INFN, Sezione di Milano. 
namical semigroups satisfying the quantum detailed balance condition [11] with respect to their stationary states.

Meanwhile, Hudson and Parthasarathy $[12,13]$ developed a powerful theory of noncommutative ("quantum") stochastic calculus, generalizing the classical Ito calculus, and it soon became clear $[13,14]$ that this theory could provide explicit constructions of dilations of quantum dynamical semigroups; however, the connection with the usual framework of dilation theory (as in [6-8]) was not made explicit.

In the present paper, we show how quantum stochastic calculus allows the construction of covariant Markov $W^{*}$-stochastic processes which are unitary dilations of the associated dynamical semigroups: the results are based on a slight generalization of Kümmerer's framework (emphasizing "covariant Markov" rather than "stationary Markov" processes) and on the results of $[13,14]$. Since the constructive aspect is very thoroughly described by $\mathbb{H u d s o n}$ and Parthasarathy in [14], we have chosen to insist on the algebraic structure of the dilation, in the spirit of $\mathbb{K}$ ümmerer [7] and of Accardi [15], cf. also Hudson, Ion and Parthasarathy [16]. For an introduction to the theory of quantum stochastic processes and its connection with the dilation problem, the reader may consult the papers by Barnett Streater and Wilde, Dümcke, Frigerio and Gorini, Hudson and Parthasarathy, Kümmerer, Lewis and Maassen, von Waldenfels in [17]. The forthcoming Proceedings of the Second Workshop on Quantum Probability and Applications, Heidelberg, 1984 [18] will cover important recent developments, including non-Fock boson and fermion quantum Wiener processes and the associated stochastic calculus.

The structure of the paper is as follows. Section 2 contains the definition of a covariant Markov $W^{*}$-stochastic process and the discussion of its relationship with an associated dynamical semigroup. The construction of covariant Markov processes by means of covariant adapted unitary evolutions is given in Section 3, and in Section 4 we show that quantum stochastic calculus provides examples of covariant adapted unitary evolutions, allowing, in particular, to construct a covariant Markov dilation for any norm continuous dynamical semigroup. Some of these results have been independently obtained by Maassen [19], with the use of a particularly convenient representation of boson Fock quantum stochastic differential equations. We defer to a forthcoming publication (contribution in [18]) the discussion of some new results concerning the problems of stationarity, time reversal and detailed balance. 


\section{Acknowledgements}

I am grateful to L. Accardi and G. C. Lupieri for stimulating discussions, and to R. L. Hudson for sending me Ref. [14] before publication. The present version of Sections 2 and 3 of the paper owes much to a discussion with $\mathbb{B}$. Kümmerer. I also thank D. B. Applebaum and H. Maassen, who had independently obtained some of the results described in Section 4 of this paper, for very interesting discussions. Finally, $\mathbb{I}$ wish to thank the referee for valuable suggestions.

\section{§2. Covariamt Markov Processes and Semigrourps}

A dynamical semigroup on a $W^{*}$-algebra $\mathbb{B}$ is a weakly* continuous one-parameter semigroup $\left\{T_{t}: t \in R^{+}\right\}$of completely positive identity preserving normal linear maps on $B, \mathbb{T}_{0}$ being the identity map. The infinitesimal generator $\mathbb{L}$ of $\mathbb{T}_{t}$ is defined on the weakly* dense domain $D(\mathbb{L})$ of those elements $b$ of $\mathbb{B}$ for which $t^{-1}\left(\mathbb{T}_{t}(b)-b\right)$ has a limit as $t \rightarrow 0$ in the weak* topology of $B$, and $\mathbb{L}(b)$ is the value of this limit.

$\mathbb{A} W^{*}$-stochastic process [4] over a $W^{*}$-algebra $B$, indexed by a set $T$, is a triple $\left(A,\left\{j_{t}: t \in \mathbb{T}\right\}, w\right)$, where $A$ is a $W^{*}$-algebra, each $j_{t}$ is a faithful normal *-representation of $B$ into $A$, with $j_{t}\left(1_{B}\right)=1_{A}, A$ is generated by $\left\{j_{t}(B): t \in \mathbb{T}\right\}$, and $w$ is a normal state on $A$, such that the $G N S$ representation of $A$ determined by $w$ is faithful. The index set $T$ will be understood to be the real line $\mathbb{R}$, unless an explicit statement to the contrary is made.

$\mathbb{A} \mathbb{W}^{*}$-stochastic process $\left(A, j_{t}, w\right)$ over $\mathbb{B}$ is said to be covariant Markov if there exists a weakly* continuous one-parameter group $\left\{\alpha_{t}: t \in \mathbb{R}\right\}$ of normal *-automorphisms of $\mathbb{A}$ such that, for each $t$ in $\mathbb{R}$,

$$
j_{t}=\alpha_{t} j_{0}
$$

and there exists a one-parameter family $\left\{E_{t]}: t \in \mathbb{R}^{+}\right\}$of normal conditional expectations in $\mathbb{A}$ such that, for $s, t$ in $\mathbb{R}^{+}$,

$$
\begin{aligned}
& \mathbb{E}_{t]}(A)=A_{t]}=\vee\left\{j_{s}(B): s \leq t\right\}, \\
& E_{s]} E_{t]}=E_{s]}: \quad s \leq t, \\
& w \circ E_{t]}=w, \\
& \alpha_{t-s} E_{s]}=E_{t]} \alpha_{t-s}, \\
& E_{0]}\left(A_{[0}\right)=A_{0},
\end{aligned}
$$


where $A_{0}=j_{0}(B)$, and where $A_{[0}=\vee\left\{j_{u}(B): u \geq 0\right\}$. The process is said to be stationary Markov if, in addition, $w=w \circ \alpha_{t}$ for all $t$ and conditional expectations satisfying (2.2)-(2.5) exist for negative $t$ as well.

If $\left(A, j_{t}, w\right)$ is a covariant Markov $W^{*}$-stochastic process over a $W^{*}$-algebra $B$, then there exists a dynamical semigroup $T_{t}$ on $B$ associated with it. Actually, this is a special case of a more general result, which we shall formulate following Accardi [15].

Let $\hat{A}$ be a $W^{*}$-algebra, let $\left\{\hat{\alpha}_{t}: t \in R\right\}$ be a weakly* continuous one-parameter group of normal *-automorphisms of $\hat{A}$, and let $\hat{w}$ be a normal state on $\hat{A}$ such that the associated GNS representation is faithful. Let $\left\{\hat{A}_{t]}: t \in R\right\}$ (resp. $\left\{\hat{A}_{[t}\right.$ : $t \in R\}$ ) be a monotonically increasing (resp. monotonically decreasing) family of $W^{*}$-subalgebras of $\hat{A}$, such that

$$
\begin{aligned}
& \hat{\alpha}_{t}\left(\hat{A}_{0]}\right) \subseteq \hat{A}_{t]}: t \in R^{+}, \\
& \hat{\alpha}_{t}\left(\hat{A}_{[0}\right) \subseteq \hat{A}_{[0}: t \in R^{+} .
\end{aligned}
$$

Let $j_{0}$ be a faithful normal *-representation of another $W^{*}$-algebra $B$ into $\hat{A}$, let $j_{t}=\hat{\alpha}_{t} j_{0}$, and suppose that

$$
j_{0}(B) \equiv A_{0} \subseteq \hat{A}_{[0} \cap \hat{A}_{0]} .
$$

Suppose that there exists a one-parameter family $\left\{\hat{E}_{t]}: t \in R^{+}\right\}$of normal conditional expectations in $\hat{A}$ satisfying the analogues of (2.2)-(2.6), with $A, \alpha_{t}$, $w, A_{t]}, E_{t]}, A_{[0}$ replaced by $\hat{A}, \hat{\alpha}_{t}, \hat{w}, \hat{A}_{t]}, \hat{E}_{t]}, \hat{A}_{[0}$ respectively. Then we have

Theorem 20 1 (Accardi [15]). Under the above assumptions, there exists a dynamical semigroup $T_{t}$ on $B$ such that

$$
\mathbb{T}_{t}(b)=j_{0}^{-1} \hat{E}_{0]} \hat{\alpha}_{t} j_{0}(b): b \in B, t \in R^{+},
$$

and, more generally,

$$
\begin{aligned}
& T_{t_{1}}\left(a_{1} T_{t_{2}-t_{1}}\left(a_{2} \cdots T_{t_{n}-t_{n-1}}\left(a_{n} b_{n}\right) \cdots b_{2}\right) b_{1}\right) \\
& \quad=j_{0}^{-1} \widehat{E}_{0]}\left(j_{t_{1}}\left(a_{1}\right) \cdots j_{t_{n}}\left(a_{n}\right) j_{t_{n}}\left(b_{n}\right) \cdots j_{t_{1}}\left(b_{1}\right)\right)
\end{aligned}
$$

for all $a_{1}, \ldots, a_{n}, b_{1}, \ldots, b_{n}$ in $B$ and for $t_{1} \leq t_{2} \leq \cdots \leq t_{n}$ in $R^{+}$,

The proof can be found in [15], or also in [4].

Remark 2.2. If $A$ is defined as the $W^{*}$-subalgebra of $\hat{A}$ generated by $j_{t}(B)$ : $t \in R$, then $\left\{\hat{\alpha}_{t} t \in R\right\}$ restricts to a weakly* continuous one-parameter group of normal *automorphisms $\left\{\alpha_{t}: t \in R\right\}$ of $A$, satisfying (2.1). Let $w$ be the restriction of $\hat{w}$ to $A$. Then $\left(A, j_{t}=\alpha_{t} j_{0}, w\right)$ is a $W^{*}$-stochastic process over $B$. 
Corollarry 2.3. If the state $\hat{w}$ on $\hat{A}$ is of the form

$$
\hat{w}=\rho \circ j_{0}^{-1} \widehat{E}_{0}
$$

where $\widehat{E}_{0}$ is a normal conditional expectation of $\hat{A}$ onto $A_{0}$ and $\rho$ is a normal state on $B$ such that the associated GNS representation is faithful, then (2.4) is equivalent to $\widehat{E}_{0} \hat{E}_{0]}=\hat{E}_{0}$, so that we may replace $\widehat{E}_{0]}$ by $\mathbb{E}_{0}=\widehat{E}_{0} \uparrow_{A}$ in (2.7) and (2.8) and the $W^{*}$-stochastic process $\left(A, j_{t}=\alpha_{t} j_{0}, w=\rho \circ j_{0}^{-1} \mathrm{E}_{0}\right)$ is both a unitary dilation and a stochastic dilation (in the sense of [10]) of the dynamical semigroup $T_{t}$.

Remark 2.4. In the case that $A_{t]} \subsetneq \widehat{A}_{t]}$, we are unable to prove in general that the process $\left(A, j_{t}=\alpha_{t} j_{0}, w\right)$ of Remark 2.2 is covariant Markov, since it is not obvious (and perhaps it is not true) that $\widehat{\mathbb{E}}_{t]}$ maps $A$ onto $A_{t]}$ and conditional expectations $E_{t]}$ of $A$ onto $A_{t]}$ satisfying (2.4) might (possibly) not exist. We shall come back to this point in the following Sections. Under the circumstances of Corollary 2.3, and if the process $\left(A, j_{t}=\alpha_{t} j_{0}, w=\rho \circ j_{0}^{-1} \mathbb{E}_{0}\right)$ is covariant Markov, we shall say that it is a covariant Markov dilation of $T_{t}$; if it is also a stationary Markov process, we shall say that it is a stationary Markov dilation.

The following result is related to the quantum version of the martingale problem, as formulated by Hasegawa and Streater [20]:

Theorem 2.5. Let $\left(A, j_{t}, w\right)$ be a covariani Markov process over $B$, let $\mathbb{T}_{t}$ be the associated dynamical semigroup on $B$. Then, for all $b$ in the domain $\mathbb{D}(L)$ of the infinitesimal generator $\mathbb{L}$ of $T_{t}$, the family $\left\{M(b ; t): t \in R^{+}\right\}$defined by

$$
M(b ; t)=j_{t}(b)-j_{0}(b)-\int_{0}^{t} j_{u}(L(b)) d u
$$

satisfies the martingale property

$$
\begin{aligned}
& M(b ; t) \in A_{t]}: \imath \in \mathbb{R}^{+}, \\
& \mathbb{E}_{s]}(\mathbb{M}(b ; t))=\mathbb{M}(b ; s): s \leq t \in \mathbb{R}^{+} .
\end{aligned}
$$

Proof. (2.11) is obvious from the definitions, and we have

$$
\begin{aligned}
\mathbb{E}_{s]} & (M(b ; t)-M(b ; s))=E_{s]} j_{t}(b)-j_{s}(b)-\int_{s}^{t} \mathbb{E}_{s]} j_{u}(\mathbb{L}(b)) d u \\
= & \alpha_{s}\left(E_{0]} \alpha_{t-s} j_{0}(b)-j_{0}(b)-\int_{s}^{t} \mathbb{E}_{01} \alpha_{u-s} j_{0}(\mathbb{L}(b)) d u\right) \\
& =j_{s}\left(T_{t-s}(b)-b-\int_{0}^{t-s} \mathbb{T}_{v}(\mathbb{L}(b)) d v\right)=0 ;
\end{aligned}
$$


where we have used (2.1), (2.5) and (2.7).

\section{§3. Construction of Covariant Markov Processes}

The actual construction of structures $\left(\hat{A}, \hat{\alpha}_{t}, \hat{w}, \hat{A}_{t]}, \hat{A}_{[t}, j_{0}, \hat{E}_{t]}\right)$ satisfying the assumptions of Theorem 2.1 (hence the construction of unitary stochastic dilations and of covariant Markov dilations of dynamical semigroups) can be effected by perturbing "trivial" ones by means of unitary cocycles satisfying a Markov property, as in [15], [16], [4], [7]. We need some preliminary definitions.

Definition 3.1. A $W^{*}$-stochastic process $\left(A,\left\{j_{(s, t)}:-\infty \leq s<t \leq+\infty\right\}, w\right)$, indexed by the open intervals in the real line, over a $W^{*}$-algebra $B$ is said to have stationary independent increments if, upon letting

$$
N(s, t)=j_{(s, t)}(B):-\infty \leq s<t \leq+\infty,
$$

the following conditions (i)-(iii) hold:

(i) $N(s, t) \subseteq N(r, u)$ for $r \leq s<t \leq u$;

(ii) there exists a weakly* continuous one-parameter group $\left\{\alpha_{t}^{0}: t \in R\right\}$ of (automatically normal) *-automorphisms of $A$ leaving $w$ invariant, such that

$$
\alpha_{t}^{0}(N(s, u))=N(s+t, u+t): t \in R,-\infty \leq s<u \leq+\infty ;
$$

(iii) for each $-\infty \leq s<t \leq+\infty$ there exists a normal conditional expectation $E_{(s, t)}$ of $A=N(-\infty,+\infty)$ onto $N(s, t)$, with $w \circ E_{(s, t)}=w$, such that, for all $a(t, u)$ in $N(t, u)$,

$$
\mathbb{E}_{(r, s)}(a(t, u))=w(a(t, u)) 1 \text { if }(r, s) \cap(t, u)=\varnothing .
$$

Remark 3.2. We shall never use the isomorphisms $j_{(s, t)}$ or the model $W^{*_{\text {m }}}$ algebra $B$. A family $\{N(s, t):-\infty \leq s<t \leq+\infty\}$ of mutually isomorphic $W^{*}$-algebras satisfying (i)-(iii), where $w$ is a state on $N(-\infty,+\infty)$ such that the GNS representation of $N(s, t)$ determined by (the restriction of $) w$ is faithful for all $s, t$, defines a $W^{*}$-stochastic process with stationary independent increments over (say) $N(0,1)$. With some abuse of language, we shall henceforth say that a $W^{*}$-stochastic process with stationary independent increments is a pair $(N(s, t), E)$, where $\{N(s, t):-\infty \leq s<t \leq+\infty\}$ is a two-parameter family of $W^{*}$-algebras as above, and where $E$ is the conditional expectation of $N(-\infty$, 
$+\infty$ ) onto the multiples of the identity defined by

$$
\mathbb{E}(a)=w(a) 1: a \in N(-\infty,+\infty) \text {. }
$$

Examples of $W^{*}$-stochastic processes with stationary independent increments can be given by means of the representations of the $\mathbb{C C R}$ or of the $\mathbb{C A R}$ algebra over $\mathbb{L}^{2}(R)$ determined by universally invariant quasi-free states, see the following Section.

Lemma 3.3。 Let $\mathbb{B}$ be a $\mathbb{W}^{*}$-algebra, let $\rho$ be a normal state on $\mathbb{B}$ such that the associated GNS representation is faithful, and let $(N(s, t), \mathbb{E})$ be a $W^{*}$-stochastic process with stationary independent increments. Define

$$
\begin{aligned}
& \hat{A}=B \otimes N(-\infty,+\infty), \\
& \hat{\alpha}_{t}=\mathrm{id}_{B} \otimes \alpha_{t}^{0}: t \in R, \\
& j_{0}(b)=b \otimes 1_{N(-\infty,+\infty)}: b \in B, \\
& \hat{A}_{t]}=B \otimes N(-\infty, t), \hat{A}_{[t}=\mathbb{B} \otimes N(t,+\infty): t \in \mathbb{R}, \\
& \hat{w}=\rho \circ j_{0}^{-1}\left(\mathrm{id}_{B} \otimes E\right), \\
& \hat{\mathbb{E}}_{t]}=\mathrm{id}_{B} \otimes E_{(-\infty, t)}: t \in \mathbb{R}^{+} .
\end{aligned}
$$

Then the assumptions of Theorem 2.1 hold. The associated dynamical semigroup on $\mathbb{B}$ is the trivial one: $\mathbb{T}_{t}=\mathrm{id}_{B}$ for all $t$.

Proof. Among the assumptions of Theorem 2.1, the only ones that are not immediate consequences of the definitions (3.5)-(3.10) are the analogues of (2.3), (2.5), (2.6). In order to prove them, we show preliminarly that the conditional expectations $\mathbb{E}_{(s, t)}$ of $N(-\infty,+\infty)$ onto $N(s, t)$ leaving $w$ invariant are uniquely determined. We identify $N(-\infty,+\infty)$ with its GNS representation determined by $w$, acting on a Hilbert space $F$ with cyclic vector $v$; the closed subspace $F(s, t)=\overline{N(s, t) v}$ of $\mathbb{F}$ is the GNS space of $N(s, t)$. If $\mathbb{E}_{(s, t)}$ leaves $w$ invariant, we have, for all $x, y$ in $N(-\infty,+\infty)$,

$$
\begin{aligned}
\left(\mathbb{E}_{(s, t)}(x) v, y v\right) & =w\left(\mathbb{E}_{(s, t)}\left(x^{*}\right) y\right)=w \circ E_{(s, t)}\left(E_{(s, t)}\left(x^{*}\right) y\right) \\
= & w\left(E_{(s, t)}\left(x^{*}\right) E_{(s, t)}(y)\right)=w\left(x^{*} E_{(s, t)}(y)\right)=\left(x v, \mathbb{E}_{(s, t)}(y) v\right),
\end{aligned}
$$

so that the map $x v \mapsto \mathbb{E}_{(s, t)}(x) v$ extends to the orthogonal projection $\mathbb{P}_{(s, t)}$ of $\mathbb{F}$ onto $F(s, t)$. Then we have

$$
\begin{aligned}
\mathbb{E}_{(s, t)}(x) a(s, t) v=\mathbb{E}_{(s, t)}( & x a(s, t)) v \\
& =\mathbb{P}_{(s, t)} x a(s, t) v: a(s, t) \in N(s, t),
\end{aligned}
$$


so that the action of $E_{(s, t)}(x) \in N(s, t)$ is uniquely determined on a dense set of vectors in the GNS space of $N(s, t)$; by the assumed faithfulness of the GNS representation, $E_{(s, t)}(x)$ is uniquely determined.

For $-\infty<s \leq t$, the product $E_{(-\infty, s)} E_{(-\infty, t)}$ is a conditional expectation onto $N(-\infty, s)$ leaving $w$ invariant, hence it coincides with $E_{(-\infty, s)}$ and the analogue of (2.3) holds. For all $s, t$ in $R$, the expression $\alpha_{t-s}^{0} E_{(-\infty, s)} \alpha_{-t+s}^{0}$ is a conditional expectation onto $N(-\infty, t)$ leaving $w$ invariant, so that the analogue of $(2.5)$ holds. The analogue of (2.6) reads

$$
\left(\mathrm{id}_{B} \otimes E_{(-\infty, 0)}\right)(B \otimes N(0,+\infty))=B \otimes 1_{N(-\infty,+\infty)},
$$

which holds as a consequence of (3.3).

Then the assumptions of Theorem 2.1 are satisfied. Since $\hat{\alpha}_{t}$ acts trivially on $A_{0}=B \otimes 1_{N(-\infty,+\infty)}$, the associated dynamical semigroup is the trivial one.

Defimition 3.4. Let $B$ be a $W^{*}$-algebra, let $(N(s, t), E)$ be a $W^{*}$ stochastic process with stationary independent increments, and assume that $B$ and $N(-\infty,+\infty)$ are concretely represented as von Neumann algebras of operators on Hilbert spaces $H_{0}$ and $F$ respectively. A covariant adapted unitary evolution based on $B$ and $N(s, t)$ is a two-parameter family $\{U(s, t)$ : $s, t \in R\}$ of unitary operators on the Hilbert space $H=H_{0} \otimes F$, satisfying

$$
U(s, t) \in B \otimes N(s, t) \quad \text { for } \quad s \leq t \in R,
$$

and, for all $s, t, u$ in $R$,

$$
\begin{aligned}
& U(s, t)=U(t, s)^{*}, \\
& U(s, t) U(t, u)=U(s, u), \\
& \hat{\alpha}_{t}(U(s, u))=U(s+t, u+t)
\end{aligned}
$$

where $\hat{\alpha}_{t}$ is defined by (3.6); and such that $s \mapsto U(s, t), t \mapsto U(s, t)$ are continuous functions in the strong operator topology.

The definition given above is essentially the same as the definition of a "covariantly adapted unitary evolution", given in [16]; as we shall see in Theorem 3.5 below, $\widehat{E}_{0}=\mathrm{id}_{B} \otimes E$ plays the role of the "reducing map" of [16]. Another appropriate name for $U(s, t)$ would be "unitary Markovian cocycle", cf. [15], [4], [7]. Examples of covariant adapted unitary evolutions will be constructed in the following Section, with the aid of the noncommutative stochastic calculus of [12-14], [18]. 
The idea of Theorem 3.5 below can be already found in the "quantum Feynman-Kac" papers [15], [16]:

Theorem 3.5. Let $B, \rho, N(s, t), \mathbb{E}$ be as in Theorem 3.3, and let $U(s, t)$ be a covariant adapted unitary evolution based on $B$ and $N(s, t)$. For each $t$ in $R$, define a map $\tilde{\alpha}_{t}$ of $\hat{A}=B \otimes N(-\infty,+\infty)$ by

$$
\tilde{\alpha}_{t}(a)=\mathbb{U}(0, t) \hat{\alpha}_{t}(a) U(0, t)^{*}: a \in \widehat{A} \text {. }
$$

Then $\left\{\tilde{\alpha}_{t}: t \in \mathbb{R}\right\}$ is a weakly* continuous one-parameter group of normal *. automorphisms of $\hat{A}$, and the assumptions of Theorem 2.1 hold with $\hat{\alpha}_{t}$ replaced by $\tilde{\alpha}_{t}$. Upon letting

$$
A=\vee\left\{j_{t}(B): t \in R\right\} ; \alpha_{t}=\tilde{\alpha}_{t} \uparrow_{A} ; E_{0}=\hat{E}_{0} \uparrow_{A}\left(\hat{E}_{0}=\mathrm{id}_{B} \otimes E\right),
$$

the $W^{*}$-stochastic process $\left(A, j_{t}=\alpha_{t} j_{0}, w=\rho \circ j_{0}^{-1} \mathbb{E}_{0}\right)$ is a unitary stochastic dilation of the dynamical semigroup $\mathbb{T}_{t}$ on $B$ defined by

$$
\mathbb{T}_{t}(b)=j_{0}^{-1} E_{0} \alpha_{t} j_{0}(b)=j_{0}^{-1} \widehat{E}_{0}\left(U(0, t) b \otimes 1_{F} U(0, t)^{*}\right) .
$$

Proof. Clearly, each $\tilde{\alpha}_{t}$ is a normal *automorphism of $\hat{A}$; the group property follows from (3.13) (3.14), and the weak* continuity is a consequence of the assumed continuity properties of $U(s, t)$. By (3.5) and $(3.11), U(0, t)$ is in $\hat{A}_{[0} \cap \hat{A}_{t]}$ for all positive $t$, so that $\tilde{\alpha}_{t}$ maps $\widehat{A}_{0]}$ into $\hat{A}_{t]}$ and $\hat{A}_{[0}$ into $\hat{A}_{[0}$ for $t$ in $R^{+}$, as $\hat{\alpha}_{t}$ does. There remains to prove that, for all $a$ in $A$,

$$
\tilde{\alpha}_{t-s} \widehat{E}_{s]}(a)=\widehat{E}_{t]}\left(\tilde{\alpha}_{t-s}(a)\right): s, t \in \mathbb{R}^{+} .
$$

The left-hand side of (3.17) can be manipulated as follows:

$$
\begin{aligned}
\tilde{\alpha}_{t-s} \hat{E}_{s]}(a) & =U(0, t-s) \hat{\alpha}_{t-s} \hat{E}_{s]}(a) U(0, t-s)^{*} \\
& =U(0, t-s) \hat{E}_{t]} \hat{\alpha}_{t-s}(a) U(0, t-s)^{*},
\end{aligned}
$$

since (2.5) holds for $\hat{\alpha}_{t}$ and $\hat{E}_{i]}$. Both $s$ and $t$ are nonnegative, so that $U(0, t-s)$ and $U(0, t-s)^{*}$ are in the range of the conditional expectation $\widehat{\mathbb{E}}_{t]}$; so they can be taken inside $\widehat{E}_{t]}$ and (3.17) follows.

Theorem 3.6. Under the additional conditions that for all $-\infty \leq s<\hat{\imath}$ $<u \leq+\infty$

$$
\begin{aligned}
& N(s, t) \subseteq N(t, u)^{\prime}, \\
& N(s, u)=\{N(s, t) \cup N(t, u)\}^{\prime \prime},
\end{aligned}
$$

the conditional expectations (3.10) map $A$ onto $A_{t]}$, and the $W^{*}$-stochastic process $\left(A, j_{t}=\alpha_{t} j_{0}, w=\rho \circ j_{0}^{-1} \mathbb{E}_{0}\right)$ is a covariant Markov dilation of the $d y$. 
namical semigroup (3.16).

Proof. We use the same notations as in the proof of Lemma 3.3. By (3.18) and (3.19), for each $-\infty \leq s<t<u \leq+\infty$ there is a natural isomorphism of $F(s, t) \otimes F(t, u)$ with $F(s, u)$, where $a(s, t) v \otimes a(t, u) v$ is identified with $a(s, t) a(t, u) v$. In particular we may identify $H=H_{0} \otimes F$ with $H(-\infty, 0)$ $\otimes F(0,+\infty)$, where $H(-\infty, 0)=H_{0} \otimes F(-\infty, 0)$. Then the conditional expectation $\widehat{E}_{0]}$ of $\hat{A}$ onto $\widehat{A}_{0]}$ is determined by

$$
\begin{aligned}
\left(\psi_{1}(-\infty, 0) \otimes \phi_{1}(0,+\infty), \hat{E}_{0]}(a) \psi_{2}(-\infty, 0) \otimes \phi_{2}(0,+\infty)\right) & \\
=\left(\psi_{1}(-\infty, 0) \otimes v(0,+\infty),\right. & \left.a \psi_{2}(-\infty, 0) \otimes v(0,+\infty)\right) \\
& \left(\phi_{1}(0,+\infty), \phi_{2}(0,+\infty)\right)
\end{aligned}
$$

for all $\psi_{1}(-\infty, 0), \psi_{2}(-\infty, 0)$ in $H(-\infty, 0)$ and for all $\phi_{1}(0,+\infty), \phi_{2}(0,+\infty)$ in $F(0,+\infty)$, where $v(0,+\infty)$ is $v$ regarded as an element of $F(0,+\infty)$. The von Neumann algebra $A$ is generated by sums of products of the elements of $A_{0]} \subseteq B \otimes N(-\infty, 0)$ and the elements of $A_{[0} \subseteq B \otimes N(0,+\infty)$; since $B \otimes 1_{F}$ $\subseteq A_{0]}$, then $A$ can be generated by sums of products of (all the) elements of $A_{0}$ and (suitable) elements of $1_{H_{0}} \otimes N(0,+\infty)$, which commute with $A_{0]}$ by (3.18). Hence $A$ is contained in the weak closure of the linear span of elements of $B(H)$ of the form

$$
a(-\infty, 0) x(0,+\infty): a(-\infty, 0) \in A_{0]}, x(0,+\infty) \in 1_{H_{0}} \otimes N(0,+\infty) .
$$

By (3.20) we have

$$
\widehat{E}_{0]}(a(-\infty, 0) x(0,+\infty))=(v, x(0,+\infty) v) a(-\infty, 0) \in A_{0]},
$$

so that $\hat{E}_{0]}$ maps $A$ onto $A_{0]}$. Taking into account (3.17), we also have

$$
\widehat{E}_{t]}(A)=\alpha_{t} \hat{E}_{0]} \alpha_{-t}(A)=\alpha_{t} \hat{E}_{0]}(A)=\alpha_{t}\left(A_{0]}\right)=A_{t]}: t \in R^{+},
$$

as required. So we may restrict $\hat{E}_{t]}$ to a conditional expectation $E_{t]}$ of $A$ onto $A_{t]}$ for all $t$ in $R^{+}$, and the process is a covariant Markov dilation of $T_{t}$.

Remark 3.7. If $(N(s, t), E)$ is a $W^{*}$-stochastic process with stationary independent increments, with group of automorphisms $\alpha_{t}^{0}$, then also $(N(-t,-s), E)$ is a $W^{*}$-stochastic process with stationary independent increments, with group of automorphisms $\alpha_{-t}^{0}$; and if $U(s, t)$ is a covariant adapted unitary evolution based on $B$ and $N(s, t)$, then $U(-s,-t)$ is a covariant adapted unitary evolution based on $B$ and $N(-t,-s)$ such that $\alpha_{-t}=\left(\alpha_{t}\right)^{-1}$ is given by $U(0,-t) \alpha_{-t}^{0}(\cdot) U(0,-t)^{*}$. Then there is another dynamical semigroup $\left\{T_{-t}\right.$ : $\left.t \in R^{+}\right\}$on $B$ such that 


$$
\mathbb{T}_{-t}=j_{0}^{-1} \hat{\mathbb{E}}_{[0} \alpha_{-t} j_{0}=j_{0}^{-1} \mathbb{E}_{0} \alpha_{-t} j_{0}: t \in \mathbb{R}^{+},
$$

where $\hat{\mathbb{E}}_{[0}=\mathrm{id}_{B} \otimes \mathbb{E}_{(0,+\infty)}$; a regression relation of the form (2.8) holds, with $\widehat{E}_{0]}$ replaced by $\widehat{E}_{[0}$ (or by $E_{0}$ ), and with $t_{r}: r=1, \ldots, n$ replaced by $-t_{r}$ throughout. $\mathbb{T}_{-t}$ will be called the time reversed dynamical semigroup. It should be noted that we have

$$
T_{-t}(b)=j_{0}^{-1} \widehat{E}_{0}\left(U(0, t) * b \otimes 1_{F} U(0, t)\right),
$$

since $U(0,-t)=\hat{\alpha}_{-t}\left(U(0, t)^{*}\right)$, and $\hat{E}_{0} \circ \hat{\alpha}_{-t}=\hat{E}_{0}, \hat{\alpha}_{-t}\left(b \otimes 1_{F}\right)=b \otimes 1_{F}$ for all $b$ in $B$ and $t$ in $R^{+}$.

\section{§4. Examples (Stochastic Evolutions)}

The noncommutative stochastic calculus of Hudson and Parthasarathy $[12,13,14]$ provides a very interesting class of covariant adapted unitary evolutions. More recently, Applebaum and Hudson [21] have constructed a large class of fermion examples; see also Barnett Streater and Wilde [22], and the contributions by Accardi and Parthasarathy and by Applebaum in [18]. The present Section is essentially a translation of the results of $[13,14,18]$ into the language that we have developed in Section 3. We try to be as general as possible.

Let $\mathbb{K}$ be a separable Hilbert space, and denote by $\mathbb{L}^{2}(R ; K)$ the Hilbert space of square-integrable $K$-valued functions defined on $R$, with inner product

$$
(f, g)=\int_{R}(f(s), g(s)) d s: f, g \in \mathbb{L}^{2}(\mathbb{R} ; \mathbb{K})
$$

For all $t$ in $\mathbb{R}$, define a unitary operator $S_{t}$ on $\mathbb{L}^{2}(\mathbb{R} ; K)$ by

$$
S_{t} f(s)=f(s-t): f \in L^{2}(R ; K), s \in R ;
$$

and for all $-\infty \leq s<t \leq+\infty$, define an orthogonal projection in $\mathbb{L}^{2}(\mathbb{R} ; \mathbb{K})$ by multiplication with the indicator function $\chi_{(s, t)}$ of the interval $(s, t)$.

Let $Q$ be a self-adjoint contraction on $K$. We shall write $Q f$ for the function $s \mapsto Q f(s)$. Then $Q$ commutes with all $S_{t}$ and with all $\chi_{(s, t)}$. All these properties of $Q$ will be understood in the following.

Example $\mathbb{A}_{0} \mathbb{1}(\mathrm{CCR})$. Let $Q$ be positive with densely defined inverse $Q^{-1}$ (then $Q^{-1} \geq 1$, since $Q$ is a contraction) and denote by $M$ the dense linear manifold in $\mathbb{L}^{2}(R ; K)$ consisting of the functions $f$ with values in $D\left(Q^{-1 / 2}\right)$ such that 


$$
q(f)=\int_{R}\left\|Q^{-1 / 2} f(s)\right\|_{K}^{2} d s<+\infty .
$$

Let $(F, W, v)$ be the cyclic representation of the $\mathbb{C C R}$ over $M$, consisting of unitary operators $W(f): f \in M$ acting on the Hilbert space $F$, with cyclic vector $v$, satisfying

$$
W(f) W(g)=W(f+g) \exp [-i \operatorname{Im}(f, g)]: f, g \in \mathbb{M}
$$

and with the generating functional

$$
(v, W(f) v)=\exp [-q(f) / 2]: f \in M ;
$$

and, for $-\infty \leq s<t \leq+\infty$, let

$$
N(s, t)=\{W(f): f \in M, \operatorname{supp} f \subseteq[s, t]\}^{\prime \prime} .
$$

As is well known (see e.g. [23]), it is always possible to reduce to the case $Q=1$ by doubling the space $K$ : denote (temporarily) by $W_{Q}$ the representation with generating functional determined by (4.5), (4.3); then a possible realization of $W_{Q}$ is

$$
W_{Q}(f)=W_{1}\left(\left[\left(Q^{-1}+1\right) / 2\right]^{1 / 2} f \oplus J\left[\left(Q^{-1}-1\right) / 2\right]^{1 / 2} f\right),
$$

where $J$ is a complex conjugation on $K$, commuting with $Q$. Let also $\left\{\alpha_{t}^{0}: t \in R\right\}$ be the weakly* continuous one-parameter group of *-automorphisms of $N(-\infty,+\infty)$ defined by

$$
\left.\alpha_{t}^{0}(\mathbb{W}(f))=W\left(\mathbb{S}_{t} f\right)\right): f \in M, t \in \mathbb{R} .
$$

Define also the conditional expectations $E, E_{(s, t)}:-\infty \leq s<t \leq+\infty$ in $N(-\infty$, $+\infty)$ by $($ see $[1,24])$

$$
\begin{aligned}
& \mathbb{E}(W(f))=\exp [-q(f) / 2] 1_{F}: f \in M, \\
& \mathbb{E}_{(s, t)}(W(f))=\exp \left[-q\left(\left[1-\chi_{(s, t)}\right] f\right) / 2\right] W\left(\chi_{(s, t)} f\right): f \in M .
\end{aligned}
$$

Then conditions (i)-(iii) of Definition 3.1 hold, and $(N(s, t), E)$ is a $W^{*}$ stochastic process with stationary independent increments. Moreover, also the additional conditions (3.18), (3.19) of Theorem 3.6 hold, and the Hilbert space $F$ acquires a natural continuous tensor product structure. This is well-known in the case $Q=1$, and extends to the general case by (4.7), since $Q$ commutes with all $\chi_{(s, t)}$.

Example $4.2(C A R)$. Let $(F, a, v)$ be the cyclic representation of the CAR 
over $L^{2}(R ; K)$, consisting of bounded operators $a(f): f \in \mathbb{L}^{2}(R ; K)$ acting on the Hilbert space $F$, such that $f \mapsto a(f)$ is a conjugate-linear map and the canonical anticommutation relations

$$
[a(f), a(g)]_{+}=0,\left[a(f), a(g)^{*}\right]_{+}=(f, g) 1_{F}: f, g \in \mathbb{L}^{2}(\mathbb{R} ; \mathbb{K})
$$

hold; the unit vector $v$ is cyclic in $F$ for $\left\{a(f), a(g)^{*}: f, g \in \mathbb{L}^{2}(R ; K)\right\}^{\prime \prime}$ and

$$
\begin{array}{r}
\left(v, a\left(f_{m}\right) * \cdots a\left(f_{1}\right)^{*} a\left(g_{1}\right) \cdots a\left(g_{n}\right)\right)=\delta_{m n} \operatorname{det}\left\{\left(v, a\left(f_{i}\right)^{*} a\left(g_{j}\right) v\right)\right\}, \\
\left(v, a(f)^{*} a(g) v\right)_{F}=(g,[(1-Q) / 2] f)_{L^{2}(R ; K)} .
\end{array}
$$

For $-\infty \leq s<t \leq+\infty$, let

$$
N(s, t)=\left\{a(f), a(f)^{*}: f \in \mathbb{L}^{2}(R ; K), \operatorname{supp} f \subseteq[s, t]\right\}^{\prime \prime} .
$$

$\mathbb{I t}$ is possible to reduce to the case $Q=1$ by doubling the space $K$ and letting

$$
B_{Q}(f)=B_{1}\left([(1+Q) / 2]^{1 / 2} f \oplus J[(1-Q) / 2]^{1 / 2} f\right),
$$

where $J$ is a complex conjugation on $K$, commuting with $Q$. Define the automorphism group $\left\{\alpha_{t}^{0}: t \in R\right\}$ of $N(-\infty,+\infty)$ by

$$
\alpha_{t}^{0}(a(f))=a\left(S_{t} f\right): f \in L^{2}(\mathbb{R} ; K), t \in \mathbb{R} .
$$

Define also the conditional expectations $E, E_{(s, t)}:-\infty \leq s<t \leq+\infty$ by [26]

$$
\begin{gathered}
\mathbb{E}(x)=(v, x v) 1_{F}: x \in N(-\infty,+\infty), \\
\mathbb{E}_{(s, t)}\left(B\left(f_{1}\right) \ldots B\left(f_{n}\right)\right)=\sum \varepsilon B\left(\chi_{(s, t)} f_{j_{1}}\right) \ldots B\left(\chi_{(s, t)} f_{j_{m}}\right) \\
\left(v, B\left(\left[1-\chi_{(s, t)}\right] f_{j_{m+1}}\right) \ldots B\left(\left[1-\chi_{(s, t)}\right] f_{j_{n}}\right) v\right) 1_{F},
\end{gathered}
$$

where

$$
B(f)=a(f)+a(f)^{*}: f \in L^{2}(R ; K),
$$

and where the summation extends to the partitions of $\{1, \ldots, n\}$ into two sets $\left\{j_{1}<\cdots<j_{m}\right\},\left\{j_{m+1}<\cdots<j_{n}\right\}$, and $\varepsilon$ is the parity of the permutation $\{1, \ldots, n\}$ $\rightarrow\left\{j_{1}, \ldots, j_{n}\right\}$.

Again, conditions (i)-(iii) of Definition 3.1 hold and $(N(s, t), E)$ is a $W^{*}$ stochastic process with stationary independent increments. However, the additional condition (3.18) of Theorem 3.6 is not valid, due to the anticommutation relations $(4.11)$.

Now we use the noncommutative stochastic calculus of $[13,14]$ to construct a covariant adapted unitary evolution. Let $B$ be a von Neumann algebra 
of operators on a Hilbert space $H_{0}$, let $\left\{X_{j}: j=1,2, \ldots\right\}$ be a sequence of operators in $B$ such that

$$
\sum_{j=1}^{\infty} X_{j}^{*} X_{j}=Y
$$

converges in the strong operator topology, and let $h$ be a self-adjoint element of $B$. Consider the case $Q=1$ in the representation of the $\mathbb{C R}$ (Fock representation). Denote by $a(f), a(f)^{*}$ the annihilation and creation operators for a boson with wave-function $f$ in $M$, and choose a complete orthonormal set $\left\{k_{j}: j=1,2, \ldots\right\}$ in $K$, once and for all.

It has been shown by Hudson and Parthasarathy in [14] that, for all $t$ in $R^{+}, u$ in $H_{0}$ and $f$ in $M, \operatorname{supp} f \subseteq[0,+\infty)$, the expressions

$$
\begin{aligned}
& A_{X}(t)[u \otimes W(f) v]=\sum_{j=1}^{\infty}\left[X_{j}^{*} \otimes a\left(\chi_{[0, t]} k_{j}\right)\right][u \otimes W(f) v] \\
& \quad=i \sum_{j=1}^{\infty} \int_{0}^{t}\left(k_{j}, f(s)\right) d s X_{j}^{*} u \otimes W(f) v, \\
& A_{X}^{*}(t)[u \otimes W(f) v]=\sum_{j=1}^{\infty}\left[X_{j} \otimes a\left(\chi_{[0, t]} k_{j}\right)^{*}\right][u \otimes W(f) v]
\end{aligned}
$$

are convergent, and define mutually adjoint operators $A_{X}(t), A_{X}^{*}(t)$ in $H(0,+\infty)$ $=H_{0} \otimes F(0,+\infty)$. Then it is possible to consider the noncommutative stochastic differential equation [14]

$$
d U(t)=U(t)\left[i\left(d A_{X}(t)+d A_{X}^{*}(t)\right)+\left(i h-\frac{1}{2} Y\right) \otimes 1_{F} d t\right]
$$

with initial condition $U(0)=1$. Roughly speaking, we have

$$
d A_{X}(t)=\sum_{j=1}^{\infty} X_{j}^{*} \otimes a\left(\chi_{[t, t+d t]} k_{j}\right) .
$$

We quote without proof the following results of [14] (or also [13], in the case of a finite-dimensional $K$ ):

Theorem 4.3 (Hudson and Parthasarathy [14]). Equation (4.21), with initial condition $U(0)=1$, has a unique solution such that $U(t)$ and $U(t)^{*}$ map $H(0, t)=H_{0} \otimes F(0, t)$ into itself and satisfy

$$
U(t)^{\#}=U(t)^{\#} \uparrow_{H(0, t)} \otimes 1_{F(t,+\infty)},
$$

where $U(t)^{\#}$ denotes either $U(t)$ or $U(t)^{*}$. The solution defines a strongly continuous one-parameter family $\left\{U(t): t \in R^{+}\right\}$of unitary operators on $H$ $(0,+\infty)$, satisfying 


$$
U(t)=\gamma\left(S_{s}^{*}\right) U(s)^{*} U(s+t) \gamma\left(S_{s}\right): s, t \in R^{+},
$$

where, for each $t$ in $\mathbb{R}^{+}$,

$$
\gamma\left(S_{t}\right)[u \otimes W(f) v]=u \otimes W\left(S_{t} f\right) v: u \in H_{0}, f \in M .
$$

Moreover, if $E$ is defined as in (4.9), we have, for all $b$ in $B$ and $t$ in $R^{+}$,

$$
E\left(U(t) b \otimes 1_{F} U(t)^{*}\right)=\exp [L t](b) \otimes \mathbb{1}_{F},
$$

where $\mathbb{L}$ is given by

$$
\mathbb{L}(b)=i[h, b]+\sum_{j=1}^{\infty}\left(X_{j}^{*} b X_{j}-\frac{1}{2}\left[X_{j}^{*} X_{j}, b\right]_{+}\right): b \in \mathbb{B} .
$$

Note that (4.27) is the general form for the generator of a norm continuous dynamical semigroup (Lindblad [27], Christensẹn and Evans [28]).

We extend $U(t)$ to $H=H_{0} \otimes F(-\infty,+\infty)$ by letting

$$
U(t)=1_{F(-\infty, 0)} \otimes U(t): t \in R^{+}
$$

(recall that $F(-\infty,+\infty)$ is isomorphic with $F(-\infty, 0) \otimes F(0,+\infty)$, by Theorem 3.6). Then $\gamma\left(S_{t}\right)$ extends to a unitary operator on $H$ satisfying $\gamma\left(S_{t}\right)$. $\cdot a \gamma\left(S_{t}^{*}\right)=\hat{\alpha}_{t}(a)$ for all $a$ in $\hat{A}$, and can be defined for negative $t$ as well. Then we have

Theorem 4.4. The two-parameter family $\{U(s, t): s, t \in \mathbb{R}\}$ of unitary operators on $H$ defined by

$$
\begin{aligned}
& U(s, t)=\gamma\left(S_{s}\right) U(t-s) \gamma\left(S_{s}^{*}\right): s \leq t \in \mathbb{R}, \\
& U(s, t)=U(t, s)^{*}: s \geq t \in \mathbb{R},
\end{aligned}
$$

is a covariant adapted unitary evolution. The dynamical semigroup $\mathbb{T}_{t}$ $=\exp [\mathrm{Lt}]$ on $B$, with infinitesimal generator $\mathbb{L}$ given by (4.27), has a covariant Markov dilation, constructed by means of $U(s, t)$. The infinitesimal generator $L^{+}$of the time reversed dynamical semigroup $T_{-t}$ is given by

$$
\mathbb{L}^{+}(b)=-i[h, b]+\sum_{j=1}^{\infty}\left(X_{j}^{*} b X_{j}-\frac{1}{2}\left[X_{j}^{*} X_{j}, b\right]_{+}\right): b \in \mathbb{B} \text {. }
$$

Proof. Conditions (3.12) and (3.14) hold by definition; then it suffices to prove (3.13) for $s, t, u \geq 0$. By (4.24) and (4.29), (4.30), we have

$$
\begin{aligned}
& U(s, t)=U(s)^{*} U(t): 0 \leq s \leq t, \\
& U(s, t)=\left[U(t)^{*} U(s)\right]^{*}=U(s)^{*} U(t): 0 \leq t \leq s ;
\end{aligned}
$$

so that, for $s, t, u \geq 0$, 


$$
U(s, t) U(t, u)=U(s)^{*} U(t) U(t) * U(u)=U(s) * U(u)=U(s, u) .
$$

It follows from the construction of [14] that (4.23) can be strengthened to $U(t-s)$ $\in B \otimes N(0, t-s)$ for $s \leq t$, then, for $s \leq t \in R$,

$$
U(s, t) \in \gamma\left(S_{s}\right) B \otimes N(0, t-s) \gamma\left(S_{s}^{*}\right)=B \otimes N(s, t),
$$

which proves (3.11). The required continuity properties follow from the strong continuity of $s \mapsto \gamma\left(S_{s}\right)$ and of $t \mapsto U(t)$.

The conditions of Theorem 3.6 are satisfied, hence the $W^{*}$-stochastic process $\left(A, j_{t}=\alpha_{t} j_{0}, w=\rho \circ j_{0}^{-1} E_{0}\right)$ is a covariant Markov dilation of the associated dynamical semigroup, defined by (4.26), (4.27). Also $T_{-t}$ is a dynamical semigroup, so it suffices to calculate

$$
\left.\frac{d}{d t}\left(u, T_{-t}(b) u^{\prime}\right)\right|_{t=0} \quad \text { for } \quad u, u^{\prime} \in H_{0}, b \in B .
$$

We have, for $t \geq 0$,

$$
\left(u, T_{-t}(b) u^{\prime}\right)=\left(U(t)[u \otimes v], b \otimes 1_{F} U(t)\left[u^{\prime} \otimes v\right]\right),
$$

where we have used (3.22), (4.29). In analogy with Theorem 7.3 of [14], we have, from Equation (4.8) of [14] and (4.33) above,

$$
\begin{aligned}
\frac{d}{d t}\left(U(t)[u \otimes v], b \otimes 1_{F} U(t)\left[u^{\prime} \otimes v\right]\right) \\
=\left([u \otimes v], L^{+}\left(U(t)^{*} b \otimes 1_{F} U(t)\right)\left[u^{\prime} \otimes v\right]\right),
\end{aligned}
$$

hence

$$
\left.\frac{d}{d t}\left(u, T_{-t}(b) u^{\prime}\right)\right|_{t=0}=\left(u, L^{+}(b) u^{\prime}\right): u, u^{\prime} \in H_{0}, b \in B
$$

which proves that $T_{-t}=\exp L^{+} t$.

Remark 4.5. The case of non-Fock quantum Brownian motion (Hudson and Lindsay [29]) may be treated by letting $Q \neq 1$, choosing $k_{j}: j=1,2, \ldots$ in (4.20) to be eigenvectors of $Q$ :

$$
Q k_{j}=\tanh \left(\beta_{j} / 2\right) k_{j}: j=1,2, \ldots,
$$

and taking, in place of $Y$ in (4.21),

$$
Y_{Q}=\sum_{j=1}^{\infty}\left(1-e^{-\beta_{j}}\right)^{-1} X_{j}^{*} X_{j}+\left(e^{\beta_{j}}-1\right)^{-1} X_{j} X_{j}^{*}
$$

the resulting generator of $T_{t}$ turns out to be [29] 


$$
\begin{aligned}
\mathbb{L}_{Q}(b)=i[h, b]+ & \sum_{j=1}^{\infty}\left\{\left(1-e^{-\beta_{j}}\right)^{-1}\left(X_{j}^{*} b X_{j}-\frac{1}{2}\left[X_{j}^{*} X_{j}, b\right]_{+}\right)\right. \\
& \left.+\left(e^{\beta_{j}}-1\right)^{-1}\left(X_{j} b X_{j}^{*}-\frac{1}{2}\left[X_{j} X_{j}^{*}, b\right]_{+}\right)\right\} .
\end{aligned}
$$

(see also Applebaum in [18]).

Remark 4.6. The stochastic differential equation (4.21) admits also of a fermion version, where the annihilation operators in (4.22) are to be interpreted as fermion annihilation operators, satisfying the canonical anticommutation relations (4.11). In [21], Applebaum and Hudson have considered only the case $\mathbb{K}=\mathbb{C}, Q=1$, and

$$
A_{X}(t)=V^{*} \theta \otimes a\left(\chi_{[0, t]}\right),
$$

where $\theta$ is a unitary involution on $H_{0}$ and $V$ anticommutes with $\theta$, but it should be clear from subsequent work of Accardi and Parthasarathy (see e.g. [18]) that also the general case (parity independent, Fock and non-Fock) can be considered (see e.g. Applebaum in [18]). Then a covariant adapteduni tary evolution can be obtained as in (4.29), (4.30), where the unitary group $\left\{\gamma\left(S_{t}\right)\right.$ : $t \in R\}$ on $H$ satisfying $\gamma\left(S_{t}\right) a \gamma\left(S_{t}^{*}\right)=\hat{\alpha}_{t}(a)$ for all $a$ in $\hat{A}$ and $t$ in $R$ is now defined by $\gamma\left(S_{t}\right) u \otimes v=u \otimes v$ and

$$
\gamma\left(S_{t}\right) u \otimes a\left(f_{1}\right)^{*} \cdots a\left(f_{n}\right)^{*} v=u \otimes a\left(S_{t} f_{1}\right)^{*} \cdots a\left(S_{i} f_{n}\right) * v
$$

for all $u$ in $H_{0}, f_{1}, \ldots, f_{n}$ in $\mathbb{L}^{2}(R ; K), n=1,2, \ldots$, and $t$ in $R$.

Due to the anticommutation relations (4.11), Theorem 3.6 cannot be applied as such. However, as pointed out to us by the referee, we may apply the formalism of $W_{2}^{*}$-algebras developed by Davies [30]: instead of tensor products we have $W_{2}^{*}$-tensor products, but again (3.20) holds and the linear span of the elements of the form (3.21) is weakly dense in $A$, so that again $\hat{E}_{t]}$ maps $A$ onto $A_{t]}$. In conclusion, we get a covariant Markov dilation also in the fermion case, in complete analogy with Theorem 4.4 .

\section{Referemces}

[1] Evans, D. E., and Lewis, J. T., Dilations of irreversible evolutions in algebraic quantum theory, Commun. Dublin Institute for Advanced Studies Ser. A., No. 24, 1977.

[2] Davies, E. B., Dilations of completely positive maps, J. London Math. Soc. (2) 17 (1978), 330-338.

[3] Lindblad, G., Non-Markovian quantum stochastic processes and their entropy, Commun. Math. Phys. 65 (1979), 281-294. 
[4] Accardi, L., Frigerio, A., and Lewis, J. T., Quantum stochastic processes, Publ. RIMS Kyoto Univ. 18 (1982), 97-113.

[ 5] Lewis, J. T., Quantum stochastic processes. I, Phys. Rep 77 (1981), 339-349.

[6] Kümmerer, B., A dilation theory for completely positive operators on $W^{*}$-algebras, Thesis, Tübingen, 1982.

[7] Kümmerer, B., A non-commutative example of a continuous Markov dilation, Semesterbericht Funktionalanalysis Tübingen, Wintersemester 1982/83 (1983), 61-91.

[ 8 ] Kümmerer, B., and Schröder, W., A Markov dilation of a non-quasifree Bloch evolution, Commun. Math. Phys. 90 (1983), 251-262.

[9] Dümcke, R., Convergence of multi-time correlations in the weak and singular coupling limits, J. Math. Phys. 24 (1983), 311-315.

[10] Frigerio, A., and Gorini, V., Markov dilations and quantum detailed balance, Commun. Math. Phys. 93 (1984), 517-532.

[11] Alicki, R., On the detailed balance condition for non-Hamiltonian systems, Rep. Math. Phys. 10 (1976), 249-258.

Kossakowski, A., Frigerio, A., Gorini, V., and Verri, M., Quantum detailed balance and KMS condition, Commun. Math. Phys. 57 (1977), 97-110.

[12] Hudson, R. L., Karandikar, R. L., and Parthasarathy, K. R., Towards a theory of noncommutative semimartingales adapted to Brownian motion and a quantum Ito's formula; and

Hudson, R. L., and Parthasarathy, K. R., Quantum diffusions. In: Theory and Applications of Random Fields, G. Kallianpur (Ed.), Lecture Notes in Control Theory and Information Sciences 49, Berlin Heidelberg New York Tokyo: Springer, 1983.

[13] Hudson, R. L., and Parthasarathy, K. R., Quantum Ito's formula and stochastic evolutions. Commun. Math. Phys. 93 (1984), 301-323.

[14] Hudson, R. L., and Parthasarathy, K. R., Stochastic dilations of uniformly continuous completely positive semigroups, Acta Math. Applicandae, 2 (I984), 353-378.

[15] Accardi, L., A quantum formulation of the Feynman-Kac formula. In: Colloquia Mathematica Societatis János Bolyai 27, Random Fields, Esztergom, 1979, pp. 25-35. - On the quantum Feynman-Kac formula, Rend. Sem. Mat. Fis. Milano 48 (1980), 135-180.

[16] Hudson, R. L., Ion, P. D. F., and Parthasarathy, K. R., Time-orthogonal unitary dilations and noncommutative Feynman-Kac formulae, Commun. Math. Phys. 83 (1982), 261-280.

[17] Accardi, L., Frigerio, A., and Gorini, V. (Eds.), Quantum Probability and Applications to the Quantum Theory of Irreversible Processes. Proceedings, Villa Mondragone, 1982, Lecture Notes in Mathematics 1055, Berlin Heidelberg New York Tokyo: Springer, 1984.

[18] Accardi, L., Van Hemmen, J. L., and von Waldenfels, W. (Editors), Proceedings of the Second Workshop on Quantum Probability and Applications, Heidelberg 1984, to appear.

[19] Maassen, H., The construction of continuous dilations by solving quantum stochastic differential equations, Semesterbericht Funktionalanalysis Tübingen, Sommersemester 84 (1984), 183-204.

[20] Hasegawa, H., and Streater, R. F., Stochastic Schrödinger and Heisenberg equations: a martingale problem in quantum stochastic processes, J. Phys. A Math. Gen. 16 (1983), L697-L703.

[21] Applebaum, D. B., and Hudson, R. L., Fermion diffusions, J. Math. Phys. 25 (1984) 858-861. 
Fermion Ito's formula and stochastic evolutions, Commun. Math. Phys., 96 (1984), 473-496.

[22] Barnett, C., Streater, R. F., and Wilde, I. F., The Itô-Clifford integral, J. Funct. Anal. 48 (1982), 172-212.

- The Itô-Clifford integral II. Stochastic differential equations, J. London Math. Soc. (2), 27 (1983), 333-384.

- The Itô-Clifford integral III. The Markov property of solutions to stochastic differential equations, Commun. Math. Phys. 89 (1983), 13-17.

[23] Manuceau, J., and Verbeure, A., Quasi-free states of the CCR algebra and Bogoliubov transformations, Commun. Math. Phys. 9, (1968), 293-302.

[24] Evans, D. E., and Lewis, J. T., Some semigroups of completely positive maps on the CCR algebra, J. Funct. Anal. 26 (1977), 369-377.

Demoen, B., Vanheuverzwijn, P., and Verbeure, A., Completely positive maps on the CCR algebra, Lett. Math. Phys. 2 (1977), 161-166.

[25] Balslev, E., Manuceau, J., and Verbeure, A., Representations of anticommutation relations and Bogoliubov transformations, Commun. Math. Phys. \& (1968), 315-326.

[26] Evans, D. E., Completely positive quasi-free maps on the CAR algebra, Commun. Math. Phys. 70 (1979), 53-68.

Fannes, M., and Rocca, F., A class of dissipative evolutions with applications in thermodynamics of fermion systems, J. Math. Phys. 21 (1980), 221-226.

[27] Lindblad, G., On the generators of quantum dynamical semigroups, Commun. Math. Phys. 48 (1976), 119-130.

[28] Christensen, E., and Evans, D. E., Cohomology of operator algebras and quantum dynamical semigroups, J. London Math. Soc. 20 (1979), 358-368.

[29] Hudson, R. L., and Lindsay, J. M., A non-commutative martingale representation theorem for non-Fock quantum Brownian motion, J. Funct. Anal., to appear.

, The classical limit of reduced quantum stochastic evolutions, preprint, 1984.

[30] Davies, E. B., Involutory automorphisms of operator algebras, Trans. Amer. Math. Soc. 158 (1971), 115-142. 
